\title{
Evaluation of Torsional Provisions in SEISMIC CoDes
}

\author{
By Anil K. Chopra, ${ }^{1}$ and Rakesh K. Goel, ${ }^{2}$
}

\begin{abstract}
The effects of plan asymmetry on the earthquake response of codedesigned, one-story systems are identified with the objective of evaluating how well these effects are represented by torsional provisions in building codes. The earthquake-induced deformations and ductility demands on resisting elements of asymmetric-plan systems, designed according to several different codes, are compared with their values if the system plan were symmetric. The presented results demonstrate that the design eccentricity in building codes should be modified in order to achicve the desirable goal of similar ductility demands on asymmetric-plan and symmetric-plan systems. The design eccentricity should be defined differently for elastic and inelastic systems; in the latter case, it should vary with the design force level or anticipated degree of inelastic action. However, it does not appear possible to reduce the additional element deformations due to plan asymmetry by modifying the design eccentricity; these deformations should be provided for in building design.
\end{abstract}

\section{INTRODUCTION}

The evaluation of torsional provisions in building codes based on computed responses of elastic, asymmetric-plan systems has been the subject of numerous studies in the past (Chandler and Hutchinson 1987; Humar 1984; Poole 1977; Rutenberg and Pekau 1983, 1987, 1989; Tso and Meng 1981). Several of these investigations have suggested a larger eccentricity, compared with current codes, in order to reflect the dynamic amplification of the torsional response arising from plan asymmetry. However, because the effects of plan asymmetry may differ significantly between elastic and inelastic systems (Goel and Chopra 1990), these conclusions may not be directly applicable to code-designed buildings, which are expected to deform significantly beyond the yield limit during intense ground shaking. Similarly, even the results of some inelastic response studies (Esteva 1987; Irvine and Kountouris 1980; Kan and Chopra 1981) may not be applicable to codedesigned systems because the assumed plan-wise distribution of structural strength is not representative of code-designed buildings, and the strength distribution can significantly influence inelastic structural response (Goel and Chopra 1990).

The inelastic response of systems with plan-wise strength distribution representative of code-designed buildings was a subject of two recent investigations that reached different conclusions (Rutenberg et al. 1989, Tso and Hongshan 1990). In one of these investigations (Tso and Hongshan 1990), the strength eccentricity of code-designed systems was determined to be approximately zero even if their stiffness eccentricity is large, and the ductility demands on the resisting elements of asymmetric-plan systems were

'Prof, , Dept. of Civ. Engrg., Univ. of California, Berkeley, CA 94720.

${ }^{2}$ Visiting Asst. Res. Engr., Dept. of Civ. Engrg., Univ. of California, Berkeley, CA 94720 . 
shown to be about the same as if the plan were symmetric. The other study (Rutenberg et al. 1989) demonstrated that the largest ductility demand among all the resisting elements may not occur in flexible-side elements, although they experience the largest deformation, but in stiff-side elements; moreover, in contrast to conclusions reached by Tso and Hongshan (1990), the peak ductility demand in asymmetric-plan systems was shown in many cases to significantly exceed that in symmetric-plan systems.

However, both investigations were concerned with the earthquake response of structural system with three resisting elements, all oriented along the direction of ground motion and none in the perpendicular directiona system that may experience significantly larger torsional motions than systems with resisting elements in each direction (Goel and Chopra 1990). Furthermore, in implementing the code design, these studies did not preclude the possibility of the design force for a stiff-side element falling below its symmetric-plan value. Because most actual buildings invariably include resisting elements in both lateral directions to provide resistance to the two horizontal components of ground motion, and because several codes and general design practice may not permit element design forces to be reduced below their symmetric-plan values, the conclusions of Rutenberg et al. (1989) and Tso and Hongshan (1990) may be restricted to the system considered and the underlying assumptions, and therefore they are not generally applicable. Obviously, there is need for more comprehensive investigation to develop a better understanding of the plan-wise distribution of strength in code-designed systems and their response behavior in order to evaluate and improve code provisions.

Aimed toward fulfilling this need, the main objective of this work is to investigate the effects of plan asymmetry on the earthquake response of code-designed, one-story systems and to determine how well these effects are represented by torsional provisions in building codes. For this purpose, we first determine how the design provisions in various codes influence the element design forces. Subsequently, the deformation and ductility demands on resisting elements of asymmetric-plan systems are compared with their values if the system plan were symmetric. Based on these results, deficiencies in code provisions are identified and improvements suggested.

\section{Torsional Provisions in Seismic Codes}

\section{Method for Computing Design Forces}

The design force $V$ specified in building codes is usually much smaller than the strength $V_{o}$ required for the system to remain elastic during intense ground shaking. Instead of computing the base shear from code formulas, which would result in different values according to different codes, the base shear is defined as

$$
V=\frac{1}{R} V_{o}
$$

where $R=$ a reduction factor that depends on the capacity of the system to safely undergo inelastic deformation during intense ground shaking. Thus, the element design forces according to various codes would differ only as a result of differences in the torsional provisions in the various codes.

In a one-story, symmetric-plan system, the design force $V$ is applied at the center of stiffness (CS). If the floor diaphragm is rigid, all resisting elements along the direction of ground motion undergo the same lateral 
displacement $u$, the lateral resisting force in the elements is $k_{j y} u$, and the total resisting force $V=K_{y} u$; in which $k_{j y}$ and $K_{y}=$ the lateral stiffness of the $j^{\text {th }}$ element and the total system, respectively. Thus, the design force in the $j^{\text {th }}$ resisting element is $\left(k_{j y} / K_{y}\right) V$, and the forces are distributed to the elements in proportion to their lateral stiffnesses or rigidities.

In asymmetric-plan systems, the design force $V$ is applied in an eccentric manner from the CS at a distance equal to design eccentricity, $e_{d}$, which is defined in the next section. Under the action of the resulting torque $e_{d} V$, the rigid roof deck will undergo rotation of $e_{d} V / K_{\theta s}$, where $K_{\theta s}=\Sigma k_{j y}$ $\left(x_{j}^{\prime}\right)^{2}+\Sigma k_{i x} y_{i}^{2}$ is the torsional stiffness about the CS, in which $x_{j}^{\prime}=x_{j} \cdot$ $e_{s} ; x_{j}=$ the distance of the $j^{\text {th }}$ element oriented in the $Y$-direction from the center of mass $(\mathrm{CM}) ; e_{s}=$ the stiffness eccentricity, i.e., the distance between the CM and the CS; and $y_{i}=$ the location of the $i^{\text {th }}$ element oriented in the $X$-direction. Thus, the design force in the $j^{\text {th }}$ element along the direction of ground motion is

$$
V_{j}=\frac{k_{j y}}{K_{y}} V+\frac{e_{d} V}{K_{0 s}}\left(-x^{\prime}{ }_{j}\right) k_{j y}
$$

The second term represents the element force associated with its deformation resulting from deck rotation and thus the change in element force due to plan asymmetry. Obviously, the torsion-induced forces are distributed to the various resisting elements in proportion to their torsional stiffnesses or rigidities.

\section{Design Eccentricity}

Most building codes require that the lateral earthquake force at each floor level of an asymmetric-plan building be applied eccentrically relative to the center of stiffness. The design eccentricity $e_{d}$ specified in most seismic codes is of the form (Earthquake Resistant 1988)

$$
\begin{aligned}
& e_{d}=\alpha e_{s}+\beta b \\
& e_{d}=\delta e_{s}-\beta b
\end{aligned}
$$

where $b=$ the plan dimension of the building perpendicular to the direction of ground motion; and $\alpha, \beta$, and $\delta=$ specified coefficients. For each element, the $e_{d}$ value leading to the larger design force is to be used. Consequently, $(3 a)$ is the design eccentricity for elements within the flexible-side of the building, and ( $3 b)$ is the design eccentricity for the stiff-side elements (Fig. 1).

The coefficients, $\alpha, \beta$ and $\delta$ vary among building codes (Earthquake Resistant 1988; "Tentative Provisions" 1978). For example, the Uniform Building Code (UBC-88) and Applied Technology Council (ATC-3) provisions specify $\beta=0.05$ and $\alpha=\delta=1$, with $\alpha=1$ implying no dynamic amplification of torsional response; the Mexico Federal District Code (MFDC77) specifies $\beta=0.1, \delta=1$, and $\alpha=1.5$, which implies dynamic amplification; the National Building Code of Canada $(N B C C-85)$ specifies $\beta=$ $0.1, \alpha=1.5$, and $\delta=0.5$; and the New Zealand Code $(N Z C-84)$ species $\beta=0.1$ and $\alpha=\delta=1$.

The first term in (3) involving $e_{s}$ is intended to account for the coupled lateral-torsional response of the building arising from lack of symmetry in plan, whereas the second term is included to consider torsional effects due to factors not explicitly considered, e.g., the rotational component of ground motion about a vertical axis, differences between computed and actual val- 


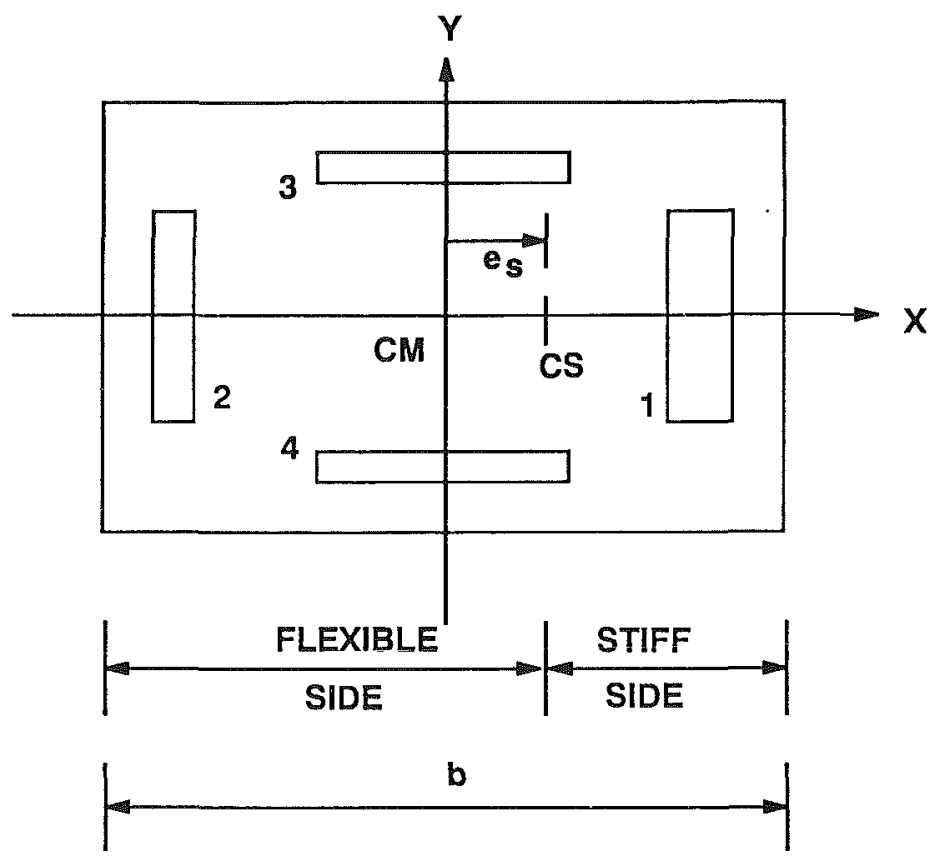

FIG. 1. Idealized One-Story System; Elements 1 and 2 Are Equidistant from CM; the Mass, Stiffness, and Strength Properties Are Symmetrical about X-Axis

ues of stiffnesses, yield strengths, and dead-load masses, and unforeseeable unfavorable distribution of live-load masses. This accidental eccentricity, $\beta b$, which is a fraction of the plan dimension, $b$, is obviously considered in design to be on either side of the CS. It is considered even in the design of symmetric-plan systems, in which case it becomes the total design eccentricity, because $e_{s}=0$.

\section{Element Design Forces}

The element design forces for an asymmetric-plan system are given by $(2)$, with the design eccentricity, $e_{d}$, defined by $(3 a)$ or $(3 b)$. The first value of $e_{d}$ will lead to the larger design force in an element in the flexible-side of the building.

$V_{j}=\left[\frac{k_{j y}}{K_{y}} V+\frac{\beta b V}{K_{\theta s}}\left(-x_{j}^{\prime}\right) k_{j y}\right]+\left[\frac{\alpha e_{s} V}{K_{\theta s}}\left(-x_{j}^{\prime}\right) k_{j y}\right] \ldots \ldots \ldots \ldots$

and the second value of $e_{d}$ results in the larger design force in stiff-side elements

$V_{j}=\left[\frac{k_{j y}}{K_{y}} V+\frac{-\beta b V}{K_{\theta s}}\left(-x^{\prime}\right) k_{j y}\right]+\left[\frac{\delta e_{s} V}{K_{\theta s}}\left(-x_{j}^{\prime}\right) k_{j y}\right] \ldots \ldots \ldots \ldots$

The first term in (4) and (5) is the element force if the system plan is symmetric $\left(e_{s}=0\right)$, and the second term arises from plan asymmetry. Thus, the element design forces for buildings with symmetrical plan are 
$V_{j o}=\frac{k_{j y}}{K_{y}} V+\frac{\beta b V}{K_{\theta s}} k_{j y}\left|x_{j}^{\prime}\right|$

wherein the first terms of (4) and (5) have been rewritten to emphasize that element forces always increases because of accidental eccentricity, with this increase being larger from codes with larger values of $\beta$. For brevity, dynamic response results are presented in this paper only for systems designed [using (4-6)] without consideration of accidental eccentricity. A parallel set of results including accidental eccentricity considerations is available in Goel and Chopra (1990).

The design force, $V_{j}$, in a resisting element of the asymmetric-plan system of Fig. 1, normalized by the design force $V_{j o}$, in the element if the system plan is symmetric, is shown in Fig. 2 for several codes. The ratio $V_{j} / V_{j o}$ also is equal to the ratio of the yield deformation of the element in asymmetricplan and symmetric-plan systems. In calculating $V_{j}$ and $V_{j o}$, the accidental eccentricity $\beta b$ is ignored. The second term, arising from plan asymmetry, is always additive for flexible-side resisting elements, (4), leading to larger design forces; this term is subtractive for stiff-side elements (5), resulting in smaller design forces because $\delta>0$ in all codes. The increase in design force for a flexible-side element grows with $e_{s}$ and the coefficient $\alpha$ in the design code (Fig. 2). Thus, among the building codes considered, NBCC85 and $M F D C-77$, which specify $\alpha=1.5$, lead to the largest increase in the design force, and $U B C-88, A T C-3$, and $N Z C-84$, which specify $\alpha=1$, result in the smallest increase. The decrease in the design force for a resisting element within the stiff side of the building grows with $e_{s}$ and the coefficient $\delta$ in the design code (Fig. 2). Thus, among the codes considered, $U B C-88$, $A T C-3, M F D C-77$, and $N Z C-84$, all of which specify $\delta=1$, lead to a decrease in the design force that is greater than from $N B C C-85$, which specifies $\delta=0.5$.

Such reduction in design forces for stiff-side elements is not permitted by several building codes, e.g., UBC-88, Peru, and India (Earthquake Resistant 1988). According to these codes, the element design forces should be in-

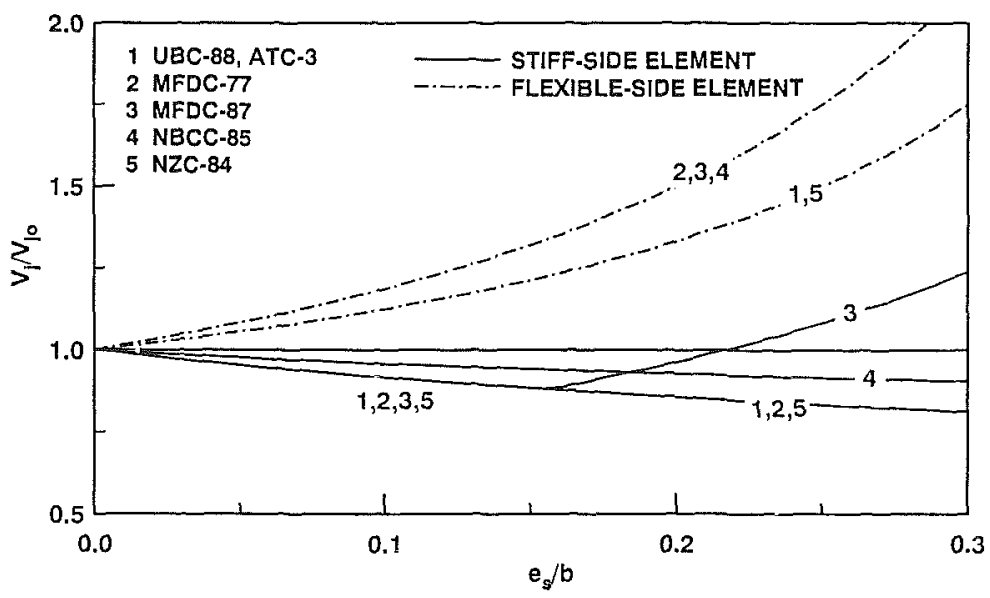

FIG. 2. Element Design Force $V_{j}$ in Asymmetric-Plan System Computed for Several Design Codes Normalized by Its Value $V_{j o}$, if System Plan is Symmetric 
creased due to plan asymmetry, but not reduced below their values for a symmetric-plan system, implying that the second term in (5) must be ignored. However, other codes and recommendations, e.g., ATC-3, MFDC$77, N B C C-85$, and $N Z C-84$, apparently do not explicitly preclude such reduction in element design forces, thus leaving open this possibility in the design process.

The 1977 Mexico Federal District Code (MFDC-77) specified the design eccentricity by (3) with $\alpha=1.5, \delta=1$, and $\beta=0.1$ (Earthquake Resistant 1988). The 1987 edition of the code, $M F D C-87$, imposes the additional requirement that element strengths shall be such that the strength eccentricity, $e_{p}$, and the stiffness eccentricity, $e_{s}$, have the same sign; $e_{p} \geq e_{s}-$ $0.2 b$ when $Q \leq 3$, and $e_{p} \geq e_{s}-0.1 b$ when $Q>3$ (Gomez and GarciaRanz 1988), where $Q$ is related to the reduction factor $R$; for medium-period and long-period systens, $Q=R$. The additional requirements of $M F D C$ 87 , if not satisfied by the element forces from $M F D C-77$, may be met by increasing the strengths of stiff-side elements, as shown in Fig. 2, or by the undesirable alternative of decreasing the strengths of flexible-side elements.

\section{Overstrength}

The total lateral design force is generally increased due to plan asymmetry because for each resisting element the more unfavorable of the two values of $e_{d}(3)$ is used to compute the design force and because some codes specify that the design force for any resisting element should not be smaller than its value if the system plan were symmetric. Buildings designed according to $U B C-88$ or $A T C-3$ possess the lowest strength, whereas those designed by $N B C C-85$ generally possess the largest strength. However, for large values of the stiffness eccentricity, systems designed by $M F D C-87$ have the largest strength because of the aforementioned additional constraint on the strength eccentricity (Goel and Chopra 1990). The strength increases with $e_{s}$ and is larger if reduction in design forces of stiff-side elements is precluded.

\section{InELASTIC Response}

Code-designed buildings typically possess a yield force much smaller than that required for this system to remain elastic during intense ground shaking. Thus, the yield force for the system is defined by (1) and the element yield forces are determined in accordance with the torsional provisions of various codes. The inelastic response of systems designed according to $U B C-88$ is investigated first and is subsequently compared with systems designed according to several other codes.

From a design point of view, it would be useful to know how the deformations and ductility demands of resisting elements in an asymmetric-plan system differ from those in the corresponding symmetric-plan system. For this purpose, presented in this investigation are the deformations $u_{i}$ and ductility demands $\mu_{i}$ of resisting elements in the asymmetric-plan system, normalized by $u_{o}$ and $\mu_{o}$, the respective response quantities of the corresponding symmetric-plan system. This is a system with $e_{s}=0$, but mass $m$, lateral stiffness $K_{y}$, torsional stiffness $K_{\mathrm{\theta s}}$ about the CS, and element stiffnesses $k_{j y}$ the same as in the asymmetric-plan system (Goel and Chopra 1990).

The normalized response quantities $u_{i} / u_{o}$ and $\mu_{i} / \mu_{o}$, for the system of Fig. 1 are presented in the form of response spectra for the first $6.3 \mathrm{sec}$ of the S00E component of the $1940 \mathrm{El}$ Centro ground motion applied in the $Y$ - 
direction. This excitation and its response spectra with various frequency regions identified are available in earlier investigations (Goel and Chopra 1990; Veletsos and Vann 1971). Because these responses are affected very little by the accidental eccentricity (Goel and Chopra 1990), it is not included in computing the design forces for the resisting elements of the asymmetricplan system and its corresponding symmetric-plan system. Two types of asymmetric-plan systems are considered: In the first system, the code design force for the stiff-side element can be smaller than the design force of the same element in the corresponding symmetric-plan system. In the second type, such a reduction is precluded. Each resisting element oriented along the ground-motion direction is idealized as elastic-perfectly lastic, with its yield force defined by the design force; the perpendicular elements are taken as elastic, an assumption that has little influence on the response (Goel and Chopra 1990). Several parameters of the system are assigned values as shown in the figures. These include the normalized stiffness eccentricity $e_{s} / r$, where $r=$ the radius of gyration of the rigid deck about the CM; ratio $\Omega=\omega_{0} /$ $\omega$ of the uncoupled torsional and lateral vibration frequencies; and the modal damping ratio $\xi$. Further, we select $\omega_{x} / \omega=1$ and $\gamma_{x}=0.5$ because the response of code-designed systems, which possess $e_{p}<<e_{s}$, is affected little by $\omega_{x} / \omega$, the ratio of the uncoupled, lateral vibration frequencies, and the ratio $\gamma_{x}$ of the torsional stiffness due to the resisting elements oriented perpendicular to the direction of ground motion to the total torsional stiffness of the system at CS. For specified values of these parameters, the locations and stiffness of the resisting elements in the system of Fig. 1 can be computed as described in Appendix B of Goel and Chopra (1990).

\section{Systems Designed by $U B C-88$}

The deformations of resisting elements in the system designed according to $U B C-88$ may be significantly affected by plan asymmetry, as indicated by the deviation of $u_{i} / u_{o}$ or $\mu_{i} / \mu_{o}$ from unity (Fig. 3). Plan asymmetry tends to reduce the deformation of the stiff-side element in medium-period, velocity-sensitive systems and increase the deformation of the flexible-side element, compared with their respective deformations in the corresponding symmetric-plan system. However, the effects of plan asymmetry on element deformations are small for short-period, acceleration-sensitive systems, and negligible for long-period, displacement-sensitive systems. The increased strength of the system, resulting from the restriction that the stiff-side element design force must not fall below its symmetric-plan value, affects the response ratio, $u_{i} / u_{o}$, in a manner consistent with the effects of strength increase on the response of single-degree-of-freedom (SDF) systems (Veletsos and V'ann 1971).

The ratio $\mu_{i} / \mu_{o}$ of the element and ductility demands in an asymmetricplan system and the corresponding symmetric-plan system also are shown in Fig. 3. If the design force for the stiff-side element is permitted to be smaller than its value in the corresponding symmetric-plan system, over a wide range of periods the element ductility demand is significantly larger due to plan asymmetry, primarily because the yield deformation of the element is smaller in asymmetric-plan systems if reduction in its design force is permitted (Fig. 2). However, if reduction in the element design force is precluded, $\mu_{i} / \mu_{o}=u_{i} / u_{o}$ because the yield deformations of this element are identical in the symmetric-plan and asymmetric-plan systems, and the previously presented observations on how deformations are affected by plan asymmetry also apply to ductility demand. The ductility demand on the 


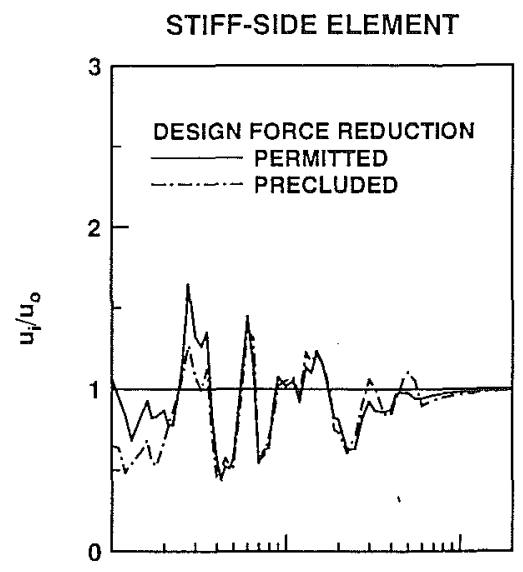

FLEXIBLE-SIDE ELEMENT
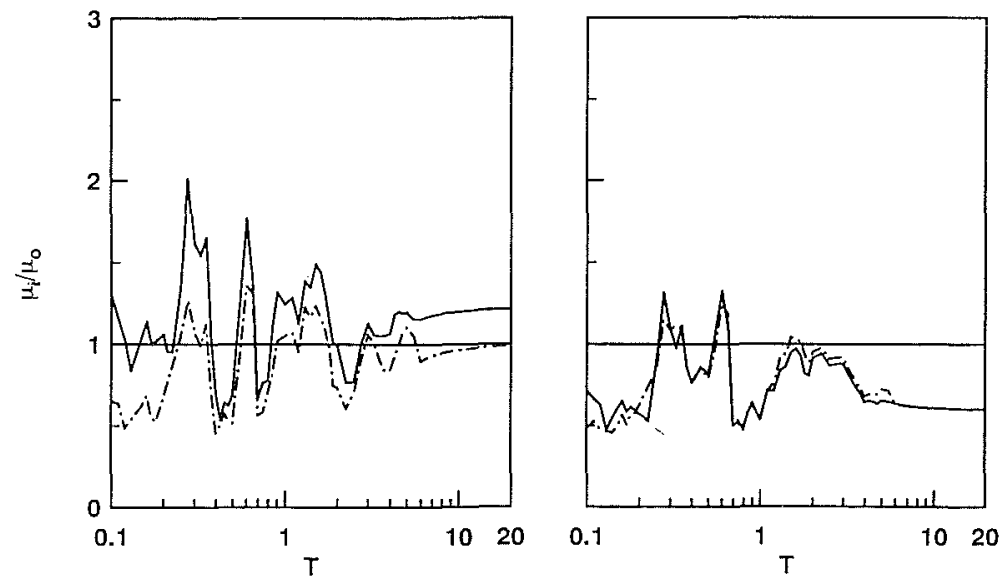

FIG. 3. Ratio of Element Deformations, $u_{i} / u_{o}$, and Ductility Demands, $\mu_{i} / \mu_{,}$, for AsymmetricPlan $\left(R=4, e_{s} / r=0.5, \Omega_{\theta}=1\right.$, and $\xi=5 \%$ ) and Corresponding Symmetric-Plan Systems Designed by UBC. 88

flexible-side element is significantly reduced because of plan asymmetry, with exceptions at few periods (Fig. 3), because the yield deformation of this element in the code-designed asymmetric-plan system is significantly larger than in the symmetric-plan system (Fig. 2). These trends are unaffected by whether the design force reduction for the stiff-side elements is permitted or not (Fig. 3), primarily because the yield deformation of the flexible-side element is unaffected by such reduction (Fig. 2).

The preceding results have demonstrated that the response of systems with and without reduction in the stiff-side element design force, arising from plan-asymmetry, may differ significantly. In particular, the ductility demand on the stiff-side element may increase significantly because of plan asymmetry when reduction in the stiff-side element design force is permitted. Since it is desirable that the element ductility demands be similar whether the plan is symmetric or not, the presented results suggest that seismic codes 
should preclude reduction in the design forces of the stiff-side elements below their values for symmetric-plan systems.

Several earlier investigations (Goel and Chopra 1990; Tso and Hongshan 1990) of the earthquake response of asymmetric-plan systems with equal stiffness and strength eccentricities, i.e., $e_{p}=e_{s}$, indicate that the largest deformation, as well as the largest ductility demand, generally occurs in the flexible-side elements, which were therefore interpreted as the most critical elements for design purposes. However, the preceding results for the system of Fig. 1 indicate that although the largest deformation among all the resisting elements of the code-designed asymmetric-plan systems for which $e_{p}$ $<<e_{s}$ occurs in the flexible-side element, the largest ductility demand may occur in the stiff-side element. Thus, additional care is required not only in the design of flexible-side elements for deformation demand, but also in the design of stiff-side elements for ductility demand; a similar observation was also made by Rutenberg et al. (1989).

\section{Systems Designed by Various Codes}

The inelastic response of the asymmetric-plan system of Fig. 1 with the element design forces determined according to various codes is compared next. The differences in the element design forces arise only from the torsional provisions in various codes because the base shear defined by (1) is identical in each case. In particular, the corresponding symmetric-plan systems are identical for all the codes with the same design base shear because the accidental eccentricity is not considered in their design. Thus, the deformation ratio $u_{i} / u_{o}$ and ductility ratio $\mu_{i} / \mu_{o}$ shown in Figs. 4 and 5 differ among systems designed by various codes because of differences in $u_{i}$ and $\mu_{i}$, whereas the responses $u_{o}$ and $\mu_{o}$ apply to all codes. Consequently, in the subsequent analysis of differences in system response designed by various codes, the response ratios and the response quantities have been used interchangeably.

The element deformation ratio $u_{i} / u_{o}$ for short-period, acceleration-sensitive and medium-period, velocity-sensitive depends on the design code; however, the response of long-period, displacement-sensitive systems is essentially independent of the design code. In the short-period spectral region, the deformation varies inversely with the strength provided by the various codes (Goel and Chopra 1990), a result that is consistent with earlier observations for SDF systems (Veletsos and Vann 1971). Thus, the system designed by $U B C-88$, which possesses the smallest strength, experiences the largest deformation, whereas the $M F D C-87$ system with the largest strength undergoes the smallest deformation, and the deformation of systems designed according to other codes falls between the two extremes (Figs. 4 and 5). The deformation of medium-period systems also decresses with increasing strength for some period values, but it may increase with strength for other period values, which again is consistent with earlier results for SDF systems. Furthermore, because the structural deformation is known to be insensitive to the strength of the system in the long-period region, the element deformations in systems designed by all the codes are essentially the same in this period region.

The trends identified herein are generally applicable to systems with strength of stiff-side elements permitted to be below that of the symmetric-plan system (Fig. 4), as well as to systems where such design force reduction is precluded (Fig. 5), except that the deformation tends to be smaller in the latter case because of increased strength, especially for short-period systems. 

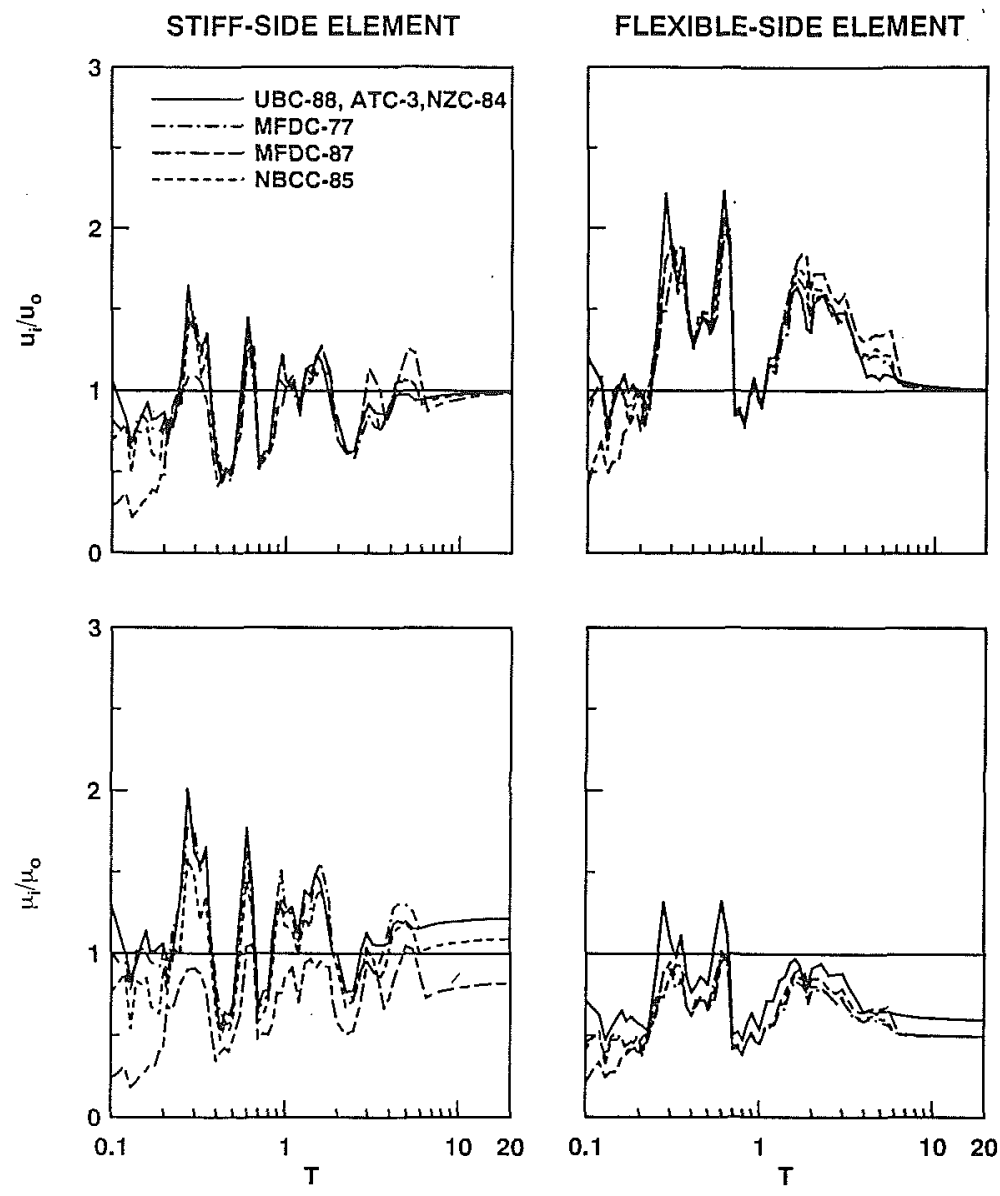

FIG. 4. Ratio of Element Deformations, $u_{i} / u_{o}$, and Ductility Demands, $\mu_{i} / \mu_{o}$, for Asymmetric-Plan ( $R=4, e_{s} / r=0.5, \Omega_{\theta}=1$, and $\left.\xi=5 \%\right)$ and Corresponding Symmetric-Plan Systems Designed by Various Codes; Reduction in Stiff-Side Element Design Force below Its Symmetric-Plan Value is Permitted

It may be noted that element deformations in systems designed according to $M F D C-77$ and $N B C C-85$ are the same in the latter case (Fig. 5) because the strengths of the two systems are identical (Goel and Chopra 1990).

It also is apparent from the results of Figs. 4 and 5 that although the deformations of resisting elements in the asymmetric-plan system depend on the design code, the differences are usually small except for $M F D C-87$. Such is the case because the strengths of various code-designed systems are not too different, except that systems designed according to $M F D C-87$ possess significantly larger strength in order to satisfy the strength eccentricity requirement, i.e., $e_{p} \geq e_{s}-0.1 b$ (Goel and Chopra 1990).

The building code by which the system is designed influences the ductility demand ratio $\mu_{i} / \mu_{o}$, and thus the ductility demand $\mu_{i}$, for the resisting elements; recall that $\mu_{o}$ is independent of the design code. The ductility 

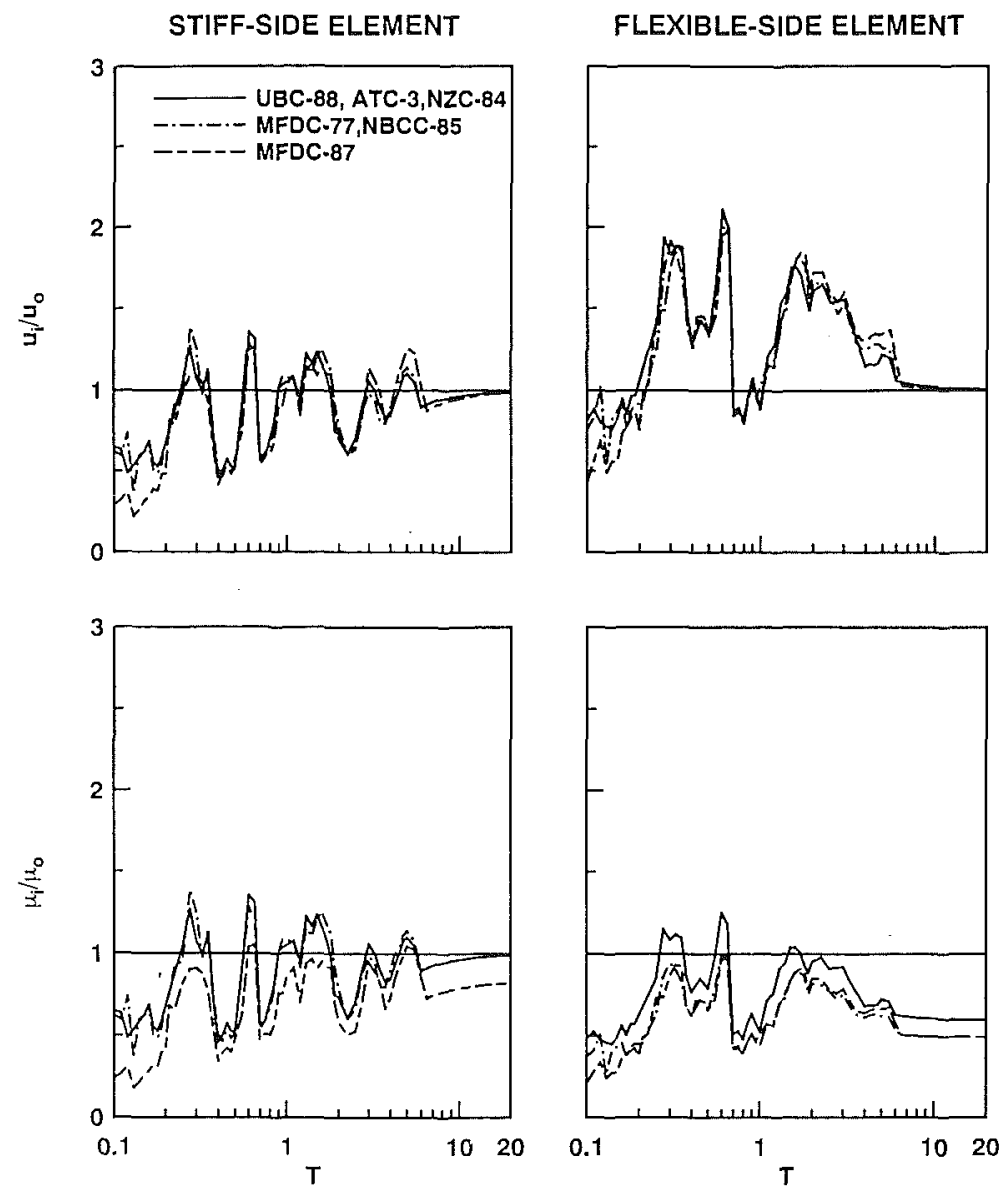

FIG. 5. Ratio of Element Deformations, $u_{i} / u_{o}$, and Ductility Demands, $\mu_{i} / \mu_{o}$, for Asymmetric Plan $\left(R=4, e_{s} / r=0.5, \Omega_{\theta}=1\right.$, and $\left.\xi=5 \%\right)$ and Corresponding Symmetric-Plan Systems Designed by Various Codes; Reduction in Stiff-Side Element Design Force Below Its Symmetric-Plan Value is Precluded

demand on a resisting element varies inversely with its yield deformation and increases proportional to the element deformation. Thus, systems designed by $U B C-88$, which possess the smallest element yield deformation (Fig. 2) and undergo the largest element deformation, especially in the shortperiod region, experience the largest ductility demand. In contrast, systems designed by $M F D C-87$ with the smallest element deformation and largest element yield deformation (Fig. 2) undergo the smallest ductility demand. Responses for systems designed by other codes fall between the two extremes (Figs. 4 and 5).

If the yield force for the stiff-side element in an asymmetric-plan system is permitted to be smaller than its symmetric-plan value, the resulting force reduction (Fig. 2) causes the ductility demand $\mu_{i}$ to be larger than its symmetric-plan value $\mu_{o}$ (Fig. 4). Among the codes considered, this increase 
in ductility demand is greatest in systems designed by $U B C-88$ and $M F D C$ 77 with $\delta=1$ (Fig. 4). If the reduction in element yield force because of plan asymmetry is precluded, i.e., $\delta=0$ in (5), the ductility demand $\mu_{i}$ on the stiff-side element in asymmetric-plan systems designed by any of the codes becomes similar to its symmetric-plan value $\mu_{o}$ over a wide range of periods (Fig. 5).

The ductility demand on the flexible-side element tends to be smaller than on the stiff-side element or on the corresponding symmetric-plan system (Figs. 4 and 5). This difference in ductility demands results primarily from the differences in element yield deformation, which is larger for the flexibleside element compared with the stiff-side element or the symmetric-plan system (Fig. 2). Codes such as NBCC-85, MFDC-77, and MFDC-87, which specify $\alpha>1$, lead to a larger increase in the yield deformation of the flexible-side element (Fig. 2), which therefore experiences smaller ductility demand compared with other codes; short-period systems designed according to $M F D C-87$ tend to experience the smallest ductility demand because the element deformation is smaller than in systems designed by other codes. The previously mentioned trends are similar in systems with design force reduction in stiff-side element (Fig. 4) and without such force reduction (Fig. 5) primarily because the yield deformation of the flexible-side element is identical in the two types of systems (Fig. 2).

The preceding results demonstrate that element deformations of systems designed according to most building codes, except $M F D C-87$, are not very different; however, the ductility demands may differ significantly among these systems. If reduction in design force of the stiff-side element below its symmetric-plan value is permitted, the $N B C C-85$-designed system has the desirable property that the ductility demand on the stiff-side element is closest, among all codes considered, to its symmetric-plan value (Fig. 4). If such design force reduction is not permitted, the ductility demands on the stiff-side element of systems designed according to all codes considered, except $M F D C-87$, are similar and close to or slightly below the symmetricplan value. In particular, the ductility demand on the stiff-side element in the $M F D C-87$-designed system tends to be significantly reduced because of plan asymmetry, suggesting that the additional requirement imposed in this code to restrict the strength eccentricity may be unnecessary.

\section{Elastic Response}

It is the intent of most seismic codes that buildings suffer no damage during some, usually unspecified, level of moderate ground shaking. Thus, the elastic response of asymmetric-plan systems designed according to several building codes is examined next.

The normalized deformation $u_{i} / u_{o}$ and ductility demand $\mu_{i}$ are presented in the form of response spectra for the El Centro ground motion; values for other parameters are fixed: $\mathcal{c}_{s} / r=0.5, R=1$, and $\xi=5 \% . R=1$ implies that the design strength $V$ of the corresponding symmetric-plan system is just sufficient for it to remain elastic during the selected excitation. However, as will be shown in subsequent sections, the code-designed, asymmetric-plan system may not remain elastic.

\section{Systems Designed by $U B C-88$}

The deformation of resisting elements in systems designed by $U B C-88$ may be significantly affected by plan asymmetry. The deformation of the 
stiff-side element is reduced because of plan asymmetry for most shortperiod, acceleration-sensitive and medium-period, velocity-sensitive systems, whereas deformation of the flexible-side element in such systems is considerably increased (Fig. 6). The element deformations of long-period, displacement-sensitive systems are essentially unaffected by plan asymmetry (Fig. 6).

The ductility demand for stiff-side and flexible-side elements in the asymmetric-plan system exceeds one in some period ranges (Fig. 6), indicating yielding in these elements, which were designed to remain elastic if the building plan were symmetric. The stiff-side element yields more if its design force is permitted to fall below its symmetric-plan value because this results in smaller yield deformation (Fig. 2). As a corollary, this element yields less if reduction in its strength is not permitted. The flexible-side element yields primarily because of its significantly larger deformation (Fig. 6) com-

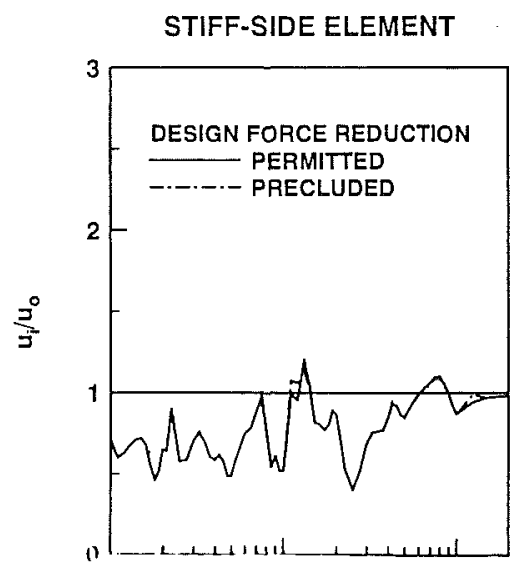

FLEXIBLE-SIDE ELEMENT
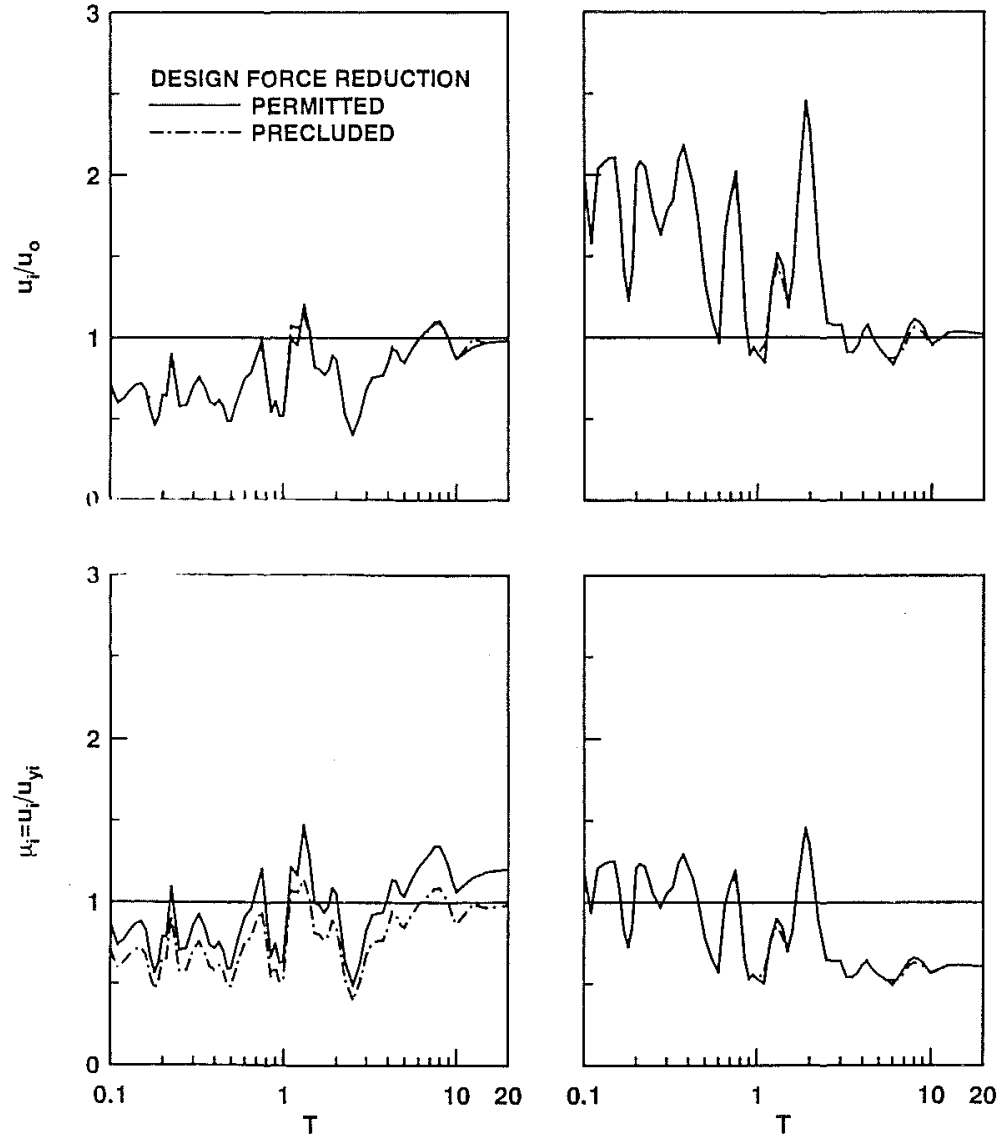

FIG. 6. Ratio of Element Deformations, $u_{i} / u_{o}$, for Asymmetric-Plan $\left(R=1, e_{s} / r=\right.$ $0.5, \Omega_{\theta}=1$, and $\xi=5 \%$ ) and Corresponding Symmetric-Plan Systems, and Element Ductility Demands $\mu_{i}$, for Asymmetric-Plan Systems Designed by UBC-88 
pared to the symmetric-plan system, although its yield deformation is also larger (Fig. 2). However, its ductility demand is unaffected whether reduction in the stiff-side element design force is permitted or not because the peak deformation as well as the yield deformation of the flexible-side element is unaffected by such reduction.

\section{Systems Designed by Various Codes}

The results of Figs. 7 and 8 show that the normalized element deformation, $u_{i} / u_{o}$, is essentially independent of the design code over a wide range of period values, and, as mentioned earlier, at each period value $u_{o}$ does not vary with the code. Therefore, the element deformations are essentially

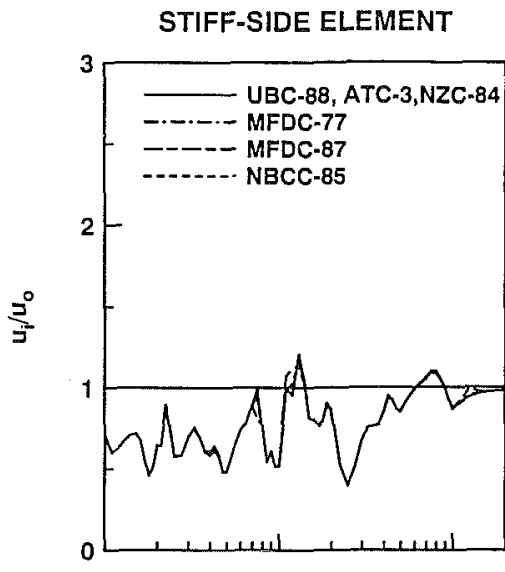

FLEXIBLE-SIDE ELEMENT
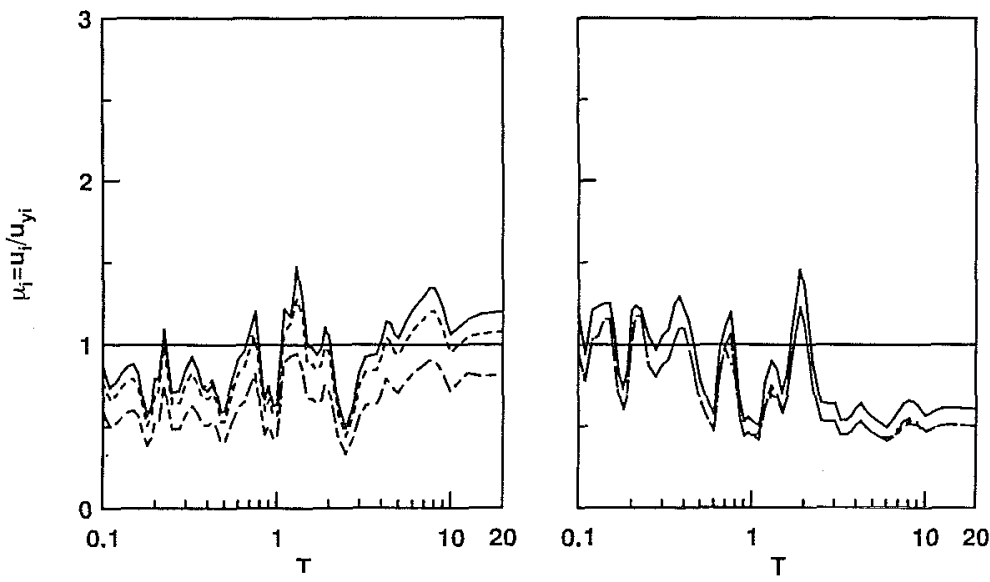

FIG. 7. Ratio of Element Deformations, $u_{i} / u_{o}$, for Asymmetric-Plan $\left(R=1, e_{s} / r=\right.$ $0.5, \Omega_{\theta}=1$, and $\xi=5 \%$ ) and Corresponding Symmetric-Plan Systems, and Element Ductility Demands $\mu_{i}$, for Asymmetric-Plan Systems Designed by Various Codes; Reduction in Stiff-Side Element Design Force below Its Symmetric-Plan Value is Permitted 

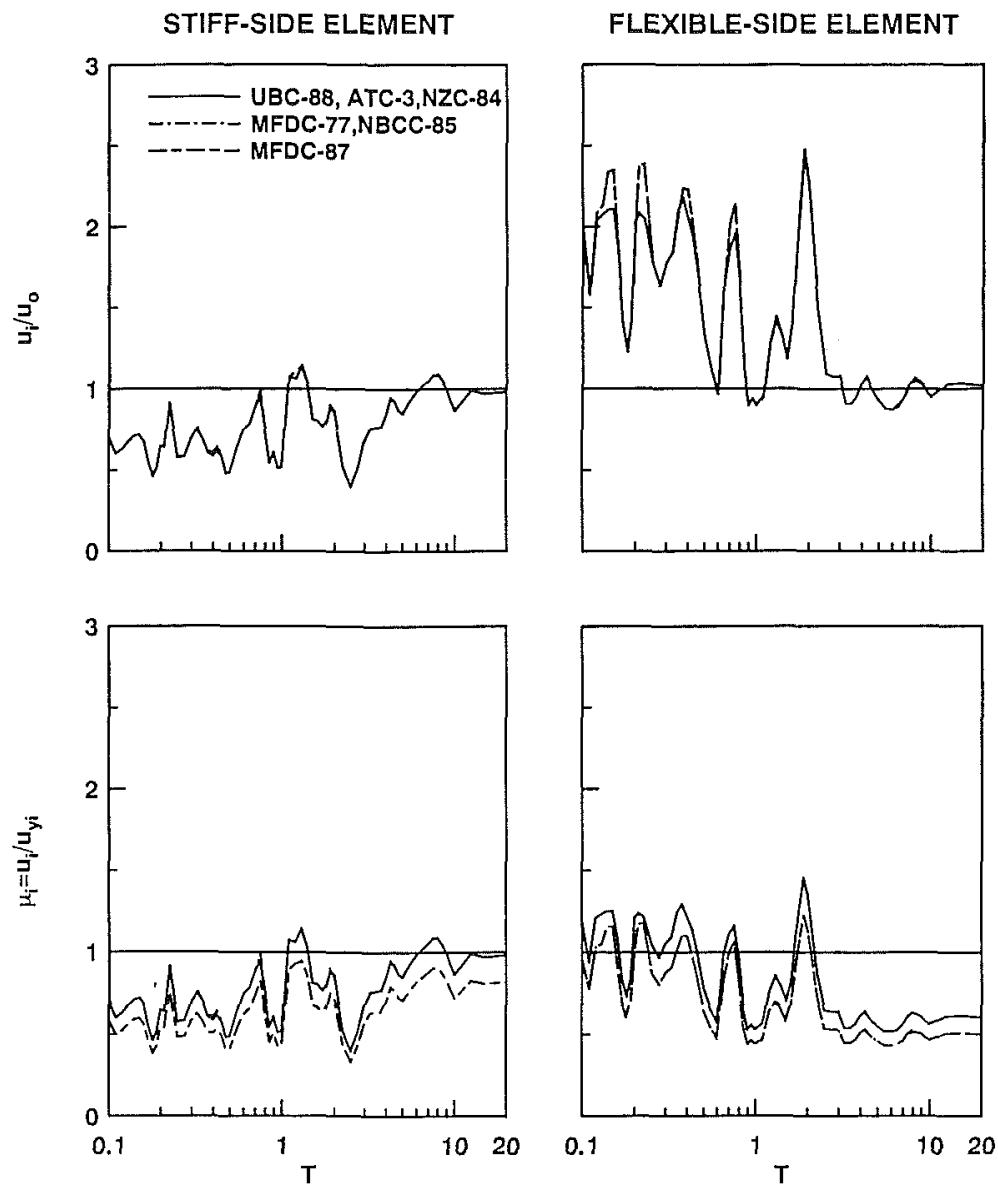

FIG. 8. Ratio of Element Deformations, $u_{i} / u_{o}$, for Asymmetric-Plan $\left(R=1, e_{s} / r=\right.$ $0.5, \Omega_{\theta}=1$, and $\xi=5 \%$ ) and Corresponding Symmetric-Plan Systems, and Element Ductility Demands, $\mu_{i}$, for Asymmetric-Plan Systems Designed by Various Codes; Reduction in Stiff-Side Element Design Force below Its Symmetric-Plan Value is Precluded

independent of the design code, which results from the fact that asymmetricplan systems designed with $R=1$ [see (1)] respond only slightly beyond the elastic range, in which case the differences in the strengths of systems designed by various codes (Goel and Chopra 1990) have very little influence on the response. These and the subsequent observations are valid regardless of whether the design force for the stiff-side element is permitted to fall below its symmetric-plan value (Figs. 7 and 8 ).

Although the element deformation $u_{i}$ in systems designed by various codes is essentially identical, the ductility demand $\mu_{i}$ may differ significantly because the ductility demand of a resisting element varies inversely with its yield deformation; note that inelastic behavior is implied when $\mu_{i}$ exceeds one, which does not happen for most period values (Figs. 7 and 8). Thus, 
systems designed by $U B C-88$, which possess the smallest element yield deformation (Fig. 2), experience the largest element ductility demands, whereas systems designed by $M F D C-87$ with the largest element yield forces undergo the smallest element ductility demands; responses of systems designed by other codes fall between these two extremes. The ductility demand on the flexible-side element is essentially the same in systems designed by $M F D C$ 77, NBCC-85, and $M F D C-87$ because the element yield deformation is identical (Fig. 2). Similarly, if the yield force of the stiff-side element is not permitted to be below its symmetric-plan value, the ductility demand on this element is the same in systems designed by $U B C-88, M F D C-77$, and NBCC-85 (Fig. 8) because the element yield deformation is identical (Fig. 2).

The preceding results demonstrate that although symmetric-plan systems with lateral yield force given by (1) with $R=1$ would remain elastic during the selected ground motion, similarly designed asymmetric-plan systems may deform into the inelastic range. Also, because of torsional motions, the element deformations may significantly exceed their deformation in the corresponding symmetric plan system. Thus, the asymmetric-plan systems may experience structural damage due to yielding and non-structural damage resulting from increased deformation.

\section{Modifications in Design Eccentricity}

The results of preceding sections indicate that deformations and ductility demands on resisting elements in a code-designed, asymmetric-plan system differ from those for the corresponding symmetric-plan system. However, it would be desirable that the responses of the two systems be similar so that the earthquake performance of the asymmetric-plan system would be similar to, and specifically no worse than, that of the symmetric-plan system. To investigate this issue further, the responses of asymmetric-plan systems, with their element yield forces computed from (4) and (5) with three different values of $\delta=1,0.5$, and 0 , are compared in Fig. 9. The first value, $\delta=1$, is typical of several codes: $U B C-88, M F D C-77$, and $N Z C-84 ; \delta=$ 0.5 is specified in $N B C C-85$; and $\delta=0$ implies no reduction in the stiffside element design force. In all cases, $\alpha=1$ and four different values of $R, 1,2,4$, and 8 , were considered (1). The ductility demand of the stiffside element is the only response quantity presented because other responses are affected very little by $\delta$. It is apparent that the ductility demand $\mu_{i}$ on the stiff-side element in the asymmetric-plan systems designed with $\delta=0$ is generally below the element ductility demand $\mu_{o}$, if the system plan were symmetric. However, for some period values, precluding reduction of stiffside element design force $(\delta=0)$ is not sufficient to keep $\mu_{i}$ below $\mu_{o}$. To achieve this objective, perhaps this design force should be increased relative to its symmetric-plan value, which implies a negative value of $\delta$ in (3); such a suggestion appeared in several earlier works on elastic systems (Pekau and Rutenberg 1987; Rutenberg and Pekau 1987, 1989).

Even if such a reduction in the stiff-side element design force is precluded, earlier inelastic response results for systems designed with $R=4$ have demonstrated that the ductility demand on the flexible-side element may be reduced because of plan asymmetry (Figs. $3-5$ ). Thus, the ductility capacity of the flexible-side element is underutilized in an asymmetric-plan system if it is designed for the ductility demand in a symmetric-plan system. To better utilize the element ductility capacity, the design eccentricity $e_{d}$ in (3a) 

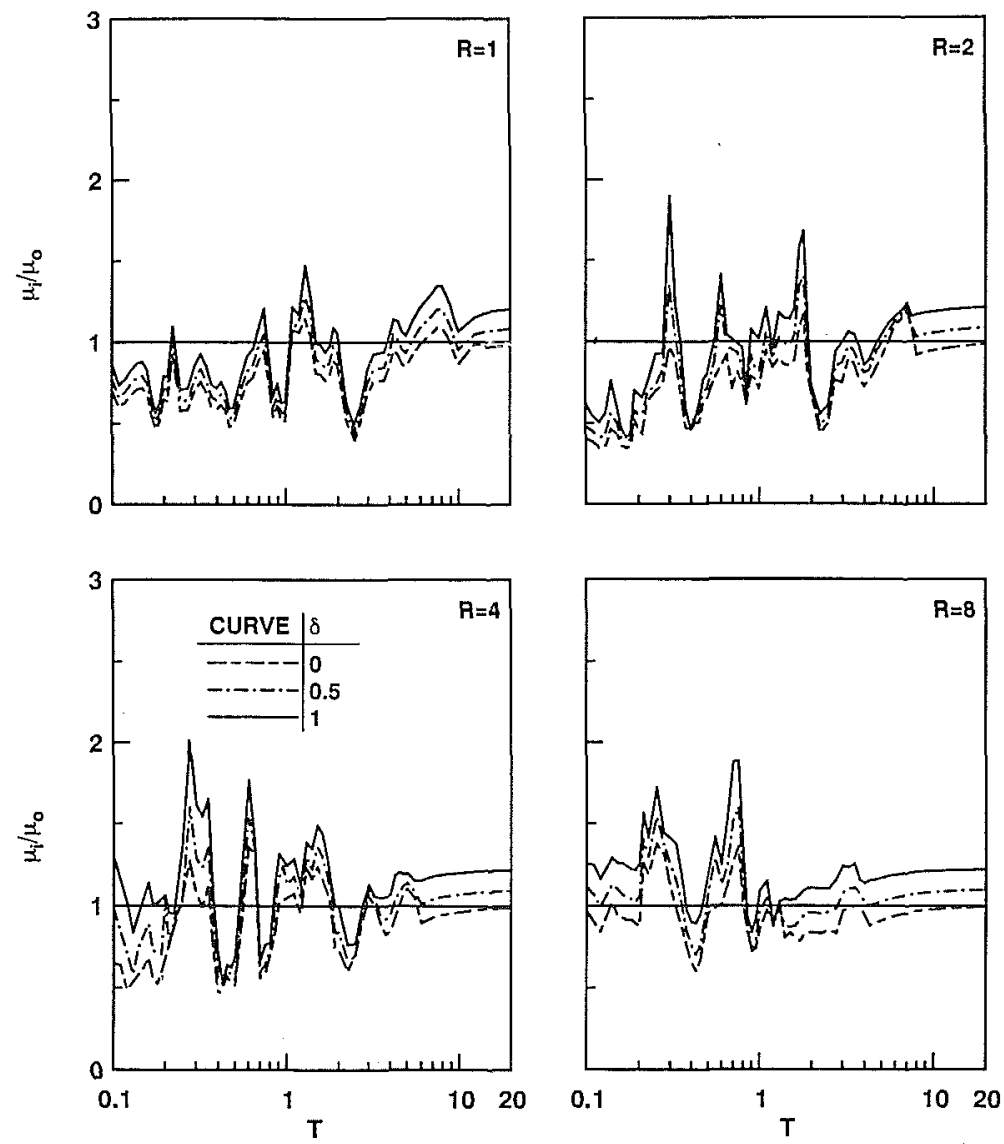

FIG. 9. Ratio of Stiff-Side Element Ductility Demands, $\mu_{i} / \mu_{o}$, for Asymmetric-Plan $\left(e_{s} / r=0.5, \Omega_{\theta}=1\right.$, and $\left.\xi=5 \%\right)$ and Corresponding Symmetric-Plan Systems; Results are Presented for Three Values of $\delta$, Fixed $\alpha=1$ and $\beta=0$, and $R=1$, 2,4 , or 8

should be modified by decreasing $\alpha$ to reduce the strength of this element. On the other hand, for systems with $R=1$, i.e., systems designed to remain elastic if their plan is symmetric and no accidental eccentricity is considered, the ductility demand on the flexible-side element in an asymmetric-plan system may exceed one indicating yielding of the element because of torsional motions (Figs. 6-8). Thus, the strength of this element should be increased by increasing $\alpha$ in (3a) to compute the design eccentricity $e_{d}$.

To further investigate these concepts, the responses of asymmetric-plan systems with their element yield forces computed from (4) and (5) with three different value of $\alpha$ are compared in Fig. 10. In addition to $\alpha=1$, two larger values are considered for systems designed with $R=1$ or 2 , two smaller values are considered when $R=8$, and one smaller and another larger value are selected when $R=4$. The ductility demand of the flexibleside element is the only response quantity presented because other response 

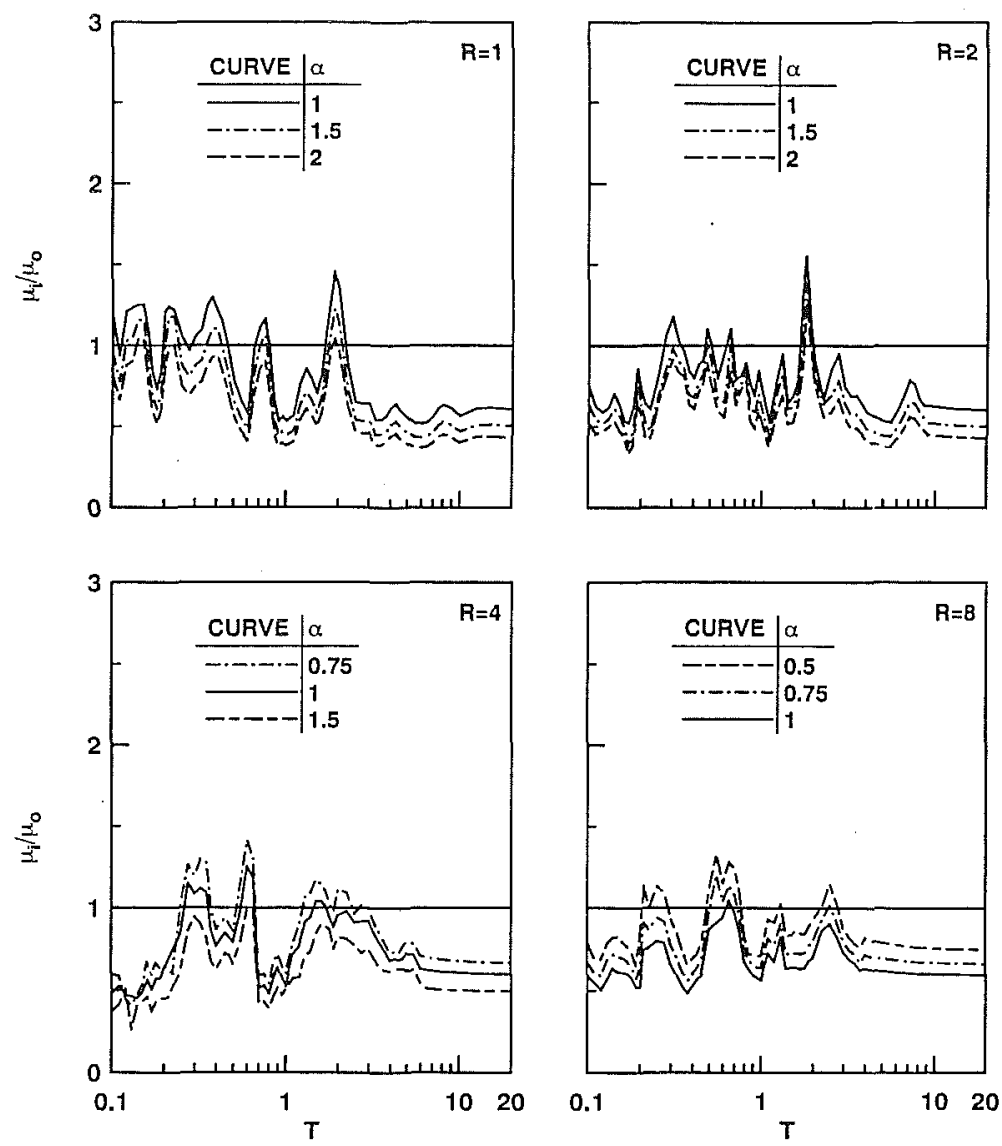

FIG. 10. Ratio of Flexible-Side Element Ductility Demands $\mu_{i} / \mu_{o}$ for AsymmetricPlan $\left(e_{s} / r=0.5, \Omega_{0}=1\right.$, and $\xi=5 \%$ ) and Corresponding Symmetric-Plan Systems. Results Are Presented for Three Values of $\alpha$, Fixed $\delta=0$ and $\beta=0$, and $R=1$, 2,4 , or 8

quantities are affected very little by $\alpha$. These results demonstrate that in order to keep the ductility demand on the flexible-side element in the asymmetric-plan system below its symmetric-plan value, $\alpha$ should be selected as follows: $\alpha=1$ if $R=8 ; \alpha=1.5$ if $R=2$ and 4 ; and $\alpha=2$ if $R=1$. However, the optimal $\alpha$-values may differ with the ground motion. Thus, response results should be generated for several ground motions to determine for code use the coefficient $\alpha$, which should depend on the design value of the reduction factor $R$.

Even if the asymmetric-plan system can be designed for significant yielding in such a way that the ductility demand on the flexible-side element does not exceed the symmetric-plan value, the element deformation may still be larger because of plan asymmetry. It may not be possible to reduce this deformation by increasing the strength of the system because, as shown by the responses of SDF systems (Veletsos and Vann 1971), the deforma- 
tion of a medium-period, velocity-sensitive system is not strongly affected by its strength and it is for such systems that the additional deformation due to plan asymmetry is most significant (Figs. 3-5). Because increasing the strength of a system beyond that required for it to remain elastic would not influence its response if it is within the elastic range, the additional deformations of elastic systems resulting from plan asymmetry also cannot be reduced. Thus, these larger deformations should be provided for in the design of asymmetric-plan structures.

\section{Dual Design Philosophy}

It is widely accepted that most buildings should be designed to: (1) Safely dissipate vibrational energy through inelastic action during intense ground shaking; and (2) remain structurally undamaged during moderate ground shaking. The first design requirement leads to the yield forces for which the structural elements should be designed to ensure that their ductility capacity is not exceeded during intense ground shaking. The second design requirement defines the strength required for the structural elements to remain elastic during moderate ground motion. Obviously, the larger of the two forces for each element is the critical design force.

The element design force in an asymmetric-plan system depends on the base shear $V$ and the design eccentricity $e_{d}(3)$. The base shear depends on the elastic spectrum and the selected reduction factor $R$ (1). As indicated by the preceding section, the design eccentricity should be defined differently for elastic and inelastic systems; in particular, the coefficient $\alpha$ and thus $e_{d}$ should increase as the reduction factor $R$ decreases. Thus, two values of the design force corresponding to the two levels of shaking should be computed for each resisting element from (4) and (5) using the appropriate values of $R$ and $\alpha$, and the critical design force should be taken as the larger of the two forces.

\section{Conclusions}

This investigation of the effects of plan asymmetry on the earthquake response of one-story systems designed by various codes, and how well these effects are represented by the torsional provisions in building codes, has led to the following conclusions.

The inelastic response of code-designed asymmetric-plan systems generally differs from that of the corresponding symmetric-plan systems. Plan asymmetry tends to increase the deformation of the flexible-side element and reduce the deformation of the stiff-side element in medium-period, velocitysensitive systems; however, the element deformations are affected little for short-period, acceleration-sensitive systems and long-period, displacementsensitive systems.

A stiff-side resisting element with design force smaller than its symmetricplan value, which is permitted by some codes, experiences increased ductility demand because of plan asymmetry. However, if the force reduction is precluded, as in some codes, the ductility demand on this element is roughly unaffected by plan asymmetry. The ductility demand on the flexible-side element in an asymmetric-plan system is significantly smaller than in the symmetric plan system, with exceptions at few periods, regardless of whether or not the design force reduction for the stiff-side element is permitted.

Although symmetric-plan systems designed with $R=1$ are expected to 
remain elastic during the design ground motion, similarly designed asymmetric-plan systems may deform into the inelastic range. Also, because of torsional motions, the element deformation may significantly exceed the deformation of the corresponding symmetric-plan system. Thus, asymmetricplan systems designed with $R=1$ may experience structural damage due to yielding and nonstructural damage resulting from increased deformations.

Building code provisions do not ensure that the deformation and ductility demands on an asymmetric-plan system are similar to those on a similarly designed symmetric-plan system. This suggests that the design eccentricity should be modified. This goal can usually be achieved for stiff-side elements by precluding any reduction in their design forces below their symmetricplan values; $\delta=0$ in the design eccentricity $e_{d}$, is equivalent to this requirement. However, for some periods values, this requirement is not sufficient and the design force for this element should be increased relative to its symmetric-plan value, which implies a negative value of $\delta$.

Similarly, the ductility demand on the flexible-side element can be kept below and close to its symmetric-plan value by modifying the coefficient $\alpha$ in the design eccentricity $e_{d}$. The optimal value of $\alpha$ in (3) depends on the design value of the reduction factor $R$ and may differ with the ground motion. Thus, response results should be generated for several ground motions to determine the coefficient $\alpha$ appropriate for use in building codes. However, it does not appear possible to reduce the additional element deformations due to plan asymmetry by modifying the design eccentricity; these large deformations should be provided for in building design.

It is widely accepted that most buildings should be designed to: (1) Safely dissipate vibrational energy though inelastic action during intense ground shaking; and (2) remain structurally undamaged during moderate ground shaking. Explicit implementation of this dual design philosophy is especially important for asymmetric-plan buildings because the design eccentricity should be defined differently for elastic and inelastic systems, and should vary with the reduction factor $R$ in the latter case. Thus, two values of the design force, corresponding to two levels of shaking, should be computed for each resisting element, using appropriate values of $R$ and $e_{d}$, and the critical design force should be taken as the larger of the two forces.

For reasons of brevity, response results presented were restricted to a single ground motion; however, a parallel set of results is available for another excitation in Goel and Chopra (1990), which also shows that the response trends are generally similar in the corresponding spectral regions of the two excitations. Thus, the conclusions derived from a single ground motion in this paper are likely to carry over to the corresponding spectral regions of other ground motions as well.

\section{ACKNOWLEDGMENTS}

This research investigation is supported by the National Science Foundation under grant $B C S-8921932$. The writers are grateful for this support.

\section{Appendix. References}

Chandler, A. M., and Hutchinson, G. L. (1987). "Evaluation of code torsional provisions by a time history approach." Earthquake Engrg. Struct. Dyn., 15 (4), $491-516$.

Esteva, L. (1987). "Earthquake engineering research and practice in Mexico after 
the 1985 earthquakes." Bull. New Zealand Nat. Soc. Earthquake Engrg., 20(3), $159-200$.

Goel, R. K. and Chopra, A. K. (1990). "Inelastic earthquake response of one-story, asymmetric-plan systems. Report No. UCB/EERC-90/14, Earthquake Engineering Research Center, University of California, Berkeley, Calif,

Gomez, R., and Garcia-Ranz, F. (1988). "The Mexico earthquake of September 19, 1985-Complementary technical norms for earthquake resistant design." Earthquake Spectra, 4(3), 441-460.

Humar, J. L. (1984). "Design for seismic torsional forces." Can. J. Civ. Engrg., $11(2), 150-163$.

International Association for Earthquake Engineering, Earthquake resistant regulations. A world list, (1988). Tokyo, Japan.

Irvine, H. M., and Kountouris, G. E. (1980). "Peak ductility demands in simple torsionally unbalanced building models subjected to earthquake ground excitation." Proc. Seventh World Conf. Earthquake Engrg., Istanbul, Turkey, 4, 117120.

Kan, C. L., and Chopra, A. K. (1981). "Torsional coupling and earthquake response of simple elastic and inelastic systems." J. Struct. Div., ASCE, 107(8), 1569-1588.

Pekau, O. A., and Rutenberg, A. (1987). "Evaluation of the torsional provisions in the 1985 NBCC." Proc. Fifth Canadian Conf. Earthquake Engrg. , Ottawa, Canada, $739-746$.

Poole, R. A. (1977). "Analysis for torsion employing provisions of NZRS 4203:1974." Bull. New Zealand Nat. Soc. Earthquake Engrg., 10(4), 219-225.

Rutenberg, A., and Pekau, O. A. (1983). "Earthquake response of asymmetric buildings: A parametric study." Proc. Fourth Canadian Conf. Earthquake Engrg., Vancouver, Canada, 271-281.

Rutenberg, A., and Pekau, O. A. (1987). "Seismic code provisions for asymmetric structures: A reevaluation." Engrg. Struct., 9(4), 255-264.

Rutenberg, A., and Pekau, O. A. (1989). "Seismic code provisions for asymmetric structures: Low period systems." Engrg. Struct., 11(2) 92-96.

Rutenberg, A., Shohet G., and Eisenberger, M. (1989). "Inelastic seismic response of code designed asymmetric structures." Fac. Pub. 303, Technion, Israel Institute of Technology, Haifa, Israel.

"Tentative provisions for the development of seismic regulations for buildings." (1978). ATC3-06, Applied Technological Council, Palo Alto, Calif.

Tso, W. K., and Hongshan, Y. (1990). "Additional seismic inelastic deformation caused by structural asymmetry." J. Earthquake Engrg. Struct. Dyn., 19(2), 243 258.

Tso, W. K., and Meng, V. (1982). "Torsional provisions in building codes." Can. J. Civ. Engrg., 9(1), 38-46.

Veletsos, A. S., and Vann, W. P. (1971). "Response of ground-excited elastoplastic systems." J. Struct. Div., ASCE, 97(4), 1257-1281. 